\title{
INNOVACIÓN SOCIAL EN ECUADOR
}

\section{SOCIAL INNOVATION IN ECUADOR}

\author{
Raúl Carpio $^{1}$, Andrea Caregua ${ }^{2}$
}

\section{Palabras clave: Innovación social, Ecuador, Responsabilidad social}

\section{Resumen}

En Ecuador, la innovación social es un tema relativamente nuevo y, dada la escasa divulgación o conocimiento de esta, no es aplicada de forma eficiente. Por ello, el objetivo del presente estudio es describir el desarrollo de la innovación social en Ecuador. Para lo cual se realizan observaciones sobre la literatura relevante y reciente en este ámbito, destacando los sectores en que se ha desarrollado con más influencia o el más utilizado por los proyectos ciudadanos. Se presentan los conceptos modernos de la innovación social, los tipos de innovación social, el proceso de la innovación social y sus sectores de aplicación. Finalmente se encontró que en Ecuador se confunden las campañas de ayuda social con innovación social, la cual corresponde a la implementación de políticas públicas en comunidades y sectores poblacionales específicos.

\section{Códigos JEL: O35, O54}

\section{Keywords:}

Social innovation, Ecuador,

Social responsibility

\begin{abstract}
In Ecuador, social innovation is a relatively new topic and, given the scant disclosure or knowledge of it, it is not applied efficiently. Therefore, the objective of this study is to describe the development of social innovation in Ecuador. For which observations are made on the relevant and recent literature in this field, highlighting the sectors in which it has been developed with the most influence or the one most used by citizen projects. Modern concepts of social innovation, types of social innovation, the process of social innovation and their sectors of application are presented. Finally, it was found that in Ecuador social aid campaigns are confused with social innovation, which corresponds to the implementation of public policies in specific communities and population sectors.
\end{abstract}

JEL Codes: O35, O54

\footnotetext{
${ }^{1}$ Universidad Espíritu Santo (UEES), (Ecuador).

E-mail: rcarpiof@uees.edu.ec

${ }^{2}$ Universidad Espíritu Santo (UEES), (Ecuador).

E-mail: acaregua@uees.edu.ec
} 



\section{INTRODUCCIÓN}

En la actualidad, la innovación social se encuentra acentuada en la responsabilidad social, ya que, para resaltar entre tantas organizaciones, es necesario presentar algo nuevo a los usuarios y, para eso, irrecusablemente es importante la innovación. Esta última se ha vuelto de gran interés por los sujetos que se encuentran en el mercado, los ámbitos pueden variar, por ejemplo, los académicos o en la dirección de empresas, para satisfacer las necesidades de algunos grupos (Nieto \& Gómez, 2004).

La innovación social se ha entendido como el campo que se alimenta de experiencias y aprendizajes personales, colectivos y organizacionales, influidos por fenómenos de cambio (Lozano, Mendoza, Felipe, \& Welter, 2016).

Así mismo, es considerada como una nueva combinación y/o nueva configuración de habilidades sociales en ciertos sitios de trabajo o de contextos sociales suscitadas por ciertos actores o constelaciones de actores. Lo anterior ocurre bajo una modalidad intencional con la meta de satisfacer o de alegar de mejor modo las necesidades y dificultades de los actores involucrados (Howaldt, 2016).

Como el tema es de gran interés ha gestionado un gran número de olas de innovación social, de manera que el mundo se encuentra en un estado cambiante cada cierto tiempo (Mazini, 2015). Precisamente, esta necesidad es lo que motiva este estudio, cuyo objetivo es describir el desarrollo de la innovación social en Ecuador. Para lo cual se revisan conceptos asociados al tema, así como procesos, métricas y aplicaciones. Estas revisiones se presentan en el marco teórico o revisión de literatura y, en consecuencia, en la sección de conclusiones se presentan los hallazgos.

\section{METODOLOGÍA}

\section{Revisión de literatura}

En español se pueden encontrar varios significados para la misma palabra, por eso es muy importante señalar el significado de las palabras (Hernández, Tirado, \& Ariza, 2016). En este estudio se ha compilado las definiciones de varios autores, permitiendo establecer una de acuerdo con el tema o término.

\section{Innovación}

Se entiende por aquel conjunto de planes, políticos, acuerdos, mecanismos sociales, formas de organización de la sociedad civil encaminados para establecer nuevos y célebres servicios, destinados a la reparación de problemas sociales en zonas muy específicas, tales como: organización política y social, en la justicia, la salud, el trabajo, la participación ciudadana, el acceso de servicios públicos, la educación, el acceso a la cultura, al descanso, a la recreación, y a un medio sano, en los ámbitos locales, regionales, nacionales o globales. Cada uno de ellos cuenta con un indicador y objetivos verificables para la transformación positiva del lugar donde se estén desarrollo (Estrada, 2014).

Pero como algunas cosas, el significado de esta palabra ha tenido evolución con el paso de los años, haciéndose más eficaz para su comprensión. Además, el interés de las personas se profundizó más detalle que hizo que las ilustraciones sean más específicas, esta evolución se presenta en la tabla 1. 


\section{TABLA 1}

\section{Evolución de diferentes conceptos de innovación social}

\begin{tabular}{cl}
\hline Autor/ año & \multicolumn{1}{c}{ Definición } \\
\hline Ogburn (1939) & $\begin{array}{l}\text { La innovación social dependerá de la invención de la época ambas van tomadas } \\
\text { de la mano en su crecimiento. }\end{array}$ \\
Christensen et. Al (2006) & $\begin{array}{l}\text { Es un subconjunto de innovaciones disruptivas con el fin principal de mejorar a la } \\
\text { sociedad. }\end{array}$ \\
Hubert (2011) & $\begin{array}{l}\text { Productos nuevos que satisfacen a las necesidades sociales. Creando nuevos } \\
\text { espacios para la acción social. }\end{array}$ \\
Blanco, Gallach \& Martínez (2015) & $\begin{array}{l}\text { Tipo de práctica de la colaboración ciudadana para relevar la crisis del mercado } \\
\text { nacional. }\end{array}$ \\
& $\begin{array}{l}\text { Proceso para hacer las cosas de modo distinto el cual tiene cuatro tipos de } \\
\text { proyectos, los cuales tienen que ser elegidos con precisión por las organizaciones. }\end{array}$ \\
& $\begin{array}{l}\text { Elementos claves que pueden ser utilizados bajo disposiciones correctas e } \\
\text { incorporen más recursos generando mecanismos que proporcionan el crecimiento } \\
\text { Marín \& Cabero (2019) }\end{array}$ \\
\hline
\end{tabular}

Se conoce que la innovación inició parcialmente en 1939 con un enfoque en los procesos mecánicos influyentes a la época. En la actualidad, las innovaciones están siendo desarrolladas en el área de las redes sociales, aunque también en el ámbito de las maquinarias que lleven consigo la tecnología actual (Rossi, 2016).

\section{Sociedad}

Son aquel conjunto de personas encargadas en el desarrollo y uso de las diversas y diferentes novedades para fortalecer y hacer ascender la economía del país. Por eso, en la actualidad, se ha identificado a la innovación como el motor de la iniciativa de todas las empresas (Martínez, González, \& Nieto, 2015).

\section{Tipos de innovación}

Anteriormente las acepciones existentes del término innovación eran referentes a las innovaciones tecnológicas o de procesos y el enfoque de estas era en el sector manufacturero. Hoy en día, el manual de Oslo propone otros tipos de innovación (ver tabla 2) cuyo fin es optimizar la producción y la productividad. Además, se conoce que estas posibilitan el incremento de la competitividad de una empresa (Echeverria, 2008).

\section{TABLA 2}

\section{Tipos de innovación}

\begin{tabular}{|c|c|c|}
\hline $\begin{array}{c}\text { Tipo de } \\
\text { innovación }\end{array}$ & Característica & $\begin{array}{l}\text { Aspectos por } \\
\text { mejorar }\end{array}$ \\
\hline $\begin{array}{l}\text { Innovación } \\
\text { Social }\end{array}$ & $\begin{array}{l}\text { Cambios } \\
\text { realizados en los } \\
\text { valores sociales. }\end{array}$ & $\begin{array}{l}\text { El bienestar, la } \\
\text { calidad de vida, la } \\
\text { inclusión social, la } \\
\text { solidaridad, la } \\
\text { participación } \\
\text { ciudadana, la } \\
\text { inclusión }\end{array}$ \\
\hline $\begin{array}{l}\text { Innovación } \\
\text { Cultural }\end{array}$ & $\begin{array}{l}\text { Cambios } \\
\text { realizados en los } \\
\text { valores culturales } \\
\text { de las personas. }\end{array}$ & $\begin{array}{l}\text { social, la atención } \\
\text { sanitaria, la calidad } \\
\text { medioambiental, la } \\
\text { eficiencia de la } \\
\text { calidad de los } \\
\text { servicios públicos }\end{array}$ \\
\hline $\begin{array}{l}\text { Innovación } \\
\text { Artística }\end{array}$ & $\begin{array}{l}\text { Cambios } \\
\text { realizados en los } \\
\text { valores artísticos } \\
\text { de las personas }\end{array}$ & $\begin{array}{l}\text { o el nivel } \\
\text { educativo de una } \\
\text { sociedad. }\end{array}$ \\
\hline
\end{tabular}

Fuente: Echeverria (2008).

En la tabla 2 se observa dos innovaciones más aparte de la social que son importantes pero complicadas de manejar, ya que no son medibles con una escala métrica explícita como el valor económico. Por ello, la innovación social tiene mayor importancia.

Otro aspecto que destaca a la innovación social entre las demás, es la variedad de campos 
en los cuales puede ser aplicada. Al respecto, se ha encontrado en orden de prioridad una lista de áreas en las que la innovación social es aplicada (Hochgerner, Franz, \& Howaldt, 2011). Tales aplicaciones se detallan en la tabla 3.

\section{TABLA 3}

\section{Aplicaciones de la innovación social}

\begin{tabular}{|c|c|c|}
\hline Aplicación & Concepto & Porcentaje \\
\hline $\begin{array}{l}\text { Innovación y } \\
\text { economía } \\
\text { social }\end{array}$ & $\begin{array}{l}\text { Anexados para } \\
\text { ampliar la función } \\
\text { económica en áreas de } \\
\text { la sociedad civil, } \\
\text { empresas y el Estado. }\end{array}$ & $33 \%$ \\
\hline $\begin{array}{l}\text { Innovaciones } \\
\text { en servicios } \\
\text { e innovación } \\
\text { social }\end{array}$ & $\begin{array}{l}\text { Integrado en los } \\
\text { conceptos de en } \\
\text { innovación social para } \\
\text { complementar los } \\
\text { servicios. }\end{array}$ & $33 \%$ \\
\hline $\begin{array}{l}\text { Innovación } \\
\text { social para } \\
\text { inclusión e } \\
\text { integración }\end{array}$ & $\begin{array}{l}\text { Promete pruebas de } \\
\text { prácticas ante las } \\
\text { innovaciones sociales. }\end{array}$ & $36 \%$ \\
\hline $\begin{array}{l}\text { Sociedad e } \\
\text { innovación } \\
\text { social }\end{array}$ & $\begin{array}{l}\text { Brindar oportunidades } \\
\text { y riesgos de } \\
\text { comunicación social } \\
\text { para habilitar la } \\
\text { innovación social. }\end{array}$ & $41 \%$ \\
\hline $\begin{array}{l}\text { Innovación } \\
\text { social y } \\
\text { negocio } \\
\text { propio }\end{array}$ & $\begin{array}{l}\text { Innovación } \\
\text { empresarial. }\end{array}$ & $42 \%$ \\
\hline $\begin{array}{l}\text { Medición } \\
\text { innovación } \\
\text { social }\end{array}$ & $\begin{array}{l}\text { Recursos sociales } \\
\text { para innovaciones } \\
\text { impacto social. }\end{array}$ & $53 \%$ \\
\hline $\begin{array}{l}\text { Innovación } \\
\text { social y } \\
\text { cambio } \\
\text { demográfico }\end{array}$ & $\begin{array}{l}\text { Obra e integración de } \\
\text { la solidaridad como } \\
\text { componentes de la } \\
\text { sociedad }\end{array}$ & $53 \%$ \\
\hline
\end{tabular}

Fuente: Hochgerner, Franz, \& Howaldt (2011)

Cabe mencionar que, siendo esta una lista ordenada de los campos de aplicación de la innovación social es posible que, dado el contexto, varíe. A su vez, los porcentajes resultantes sean alterados.

\section{Características de la innovación social}

Una de las características principales de la innovación social, es precisamente, la intervención social, ya que esta es el núcleo de las actividades dentro del ámbito de los servicios sociales. Además, representa la medida de control en el tráfico económico. Constituye una característica dado que permite tener un criterio de quienes conforman el proyecto (Fantova, 2018).

Por otro lado, cabe mencionar que, para que exista la innovación, se ha visto necesario que la economía social se vea afectada por diversas problemáticas. En ese sentido, esta también constituye una característica de la innovación social (Ávila \& Campos, 2018).

\section{Problemas que atacan la innovación social}

El mayor problema es que en la antigüedad las direcciones principales que se le daba a la innovación eran aspectos físicos, tales como: la forma, materiales, tecnología de producción, entre otros (Ortíz, 2016). Cuando en realidad la visión debería ser hacia la estimulación de conductas que promuevan la sostenibilidad, emociones y experiencias, a fin de enfrentar los retos complejos como los sociales.

\section{Sostenibilidad}

Es la clave para la vida empresarial, ya que con esto la rentabilidad del negocio se convierte en una estrategia primordial para mantener a la empresa sin fracasos y, con el tiempo, llegar a la cima empresarial (Moliner, Azorín, Guilló, Ortega, \& Gamero, 2019).

Además, las sostenibilidad se considera como un fenómeno de la globalización necesario para el desarrollo de empresas de calidad, ya que, si no es rentable, no es una buena inversión (Mora \& Martínez, 2018).

\section{Emociones}

Son las reacciones que ayudan a culminar o iniciar un deseo, esto da paso a la relación sentimental, misma que se puede tener con cosas o personas. Inclusive la forma en cómo las personas toman sus decisiones tiene que ver con el manejo de sus emociones. En consecuencia, se resalta un tema muy significativo que es la inteligencia emocional, que puede ser medible 
con el coeficiente emocional de las personas (Marin, 2018).

En específico, la inteligencia emocional se aplica infinidades de veces en la toma de decisiones en las empresas, por variedades de líderes para tener un trabajo eficaz, más que cualquier cosa es una gran estrategia para utilizar a beneficio de las instituciones (Goleman, 2018). Mientras que en los trabajadores impacta en su desempeño laboral, considerando las siguientes variables: autoconocimiento, automotivación, autocontrol y control de las relaciones interpersonales (Orué-Arias, 2017).

\section{Experiencia}

La experiencia es uno de los motores de cambio e innovación, dado que es preciso contar con alguien capacitado para que el crecimiento y la sostenibilidad del proyecto sean correspondientes a los objetivos del negocio o emprendimiento (Serrano, Hervás, \& Campos, 2017).

\section{Responsabilidad social}

Se trata de un factor que con su debida influencia puede llegar a transformar la sociedad. Para esto son utilizadas las universidades, ya que, al prestar la formación de profesionales, van construyendo una mejor comunidad. La educación tiene como fin las evaluaciones internas del establecimiento y objetivos orientados a la mejora continua (López, 2016).

\section{Contexto ecuatoriano}

Existe puntualmente un problema que deja inexistente lo antes mencionado para la innovación y es la educación. Aunque es un derecho vital de los ciudadanos, aun la economía es un factor grave a la hora de considerar el poco alcance que tiene la educación en varios lugares, más que nada en los rurales. Para la innovación del país, es necesario que la educación sea el punto primordial por considerar a la hora de invertir, porque es la única fuente de conocimiento para alcanzar el aumento de ventajas en el mundo laboral (Calderón, 2015).
Otro factor desfavorecedor en Ecuador es la pobreza, ya que causa el abandono escolar y, con esto, borra la posibilidad de que en el futuro las personas puedan innovar con los conocimientos necesarios.

La educación es un factor importante, ya que con esto se puede fortalecer la creatividad de las personas, que es un factor fundamental en la innovación. Además, es útil para la implementación de estrategias innovadoras y responsables, lo cual establece condiciones que contribuyen a que la sociedad sea mejor y denote una mayor estabilidad económica (Álvarez \& Macías, 2015).

\section{Innovación social en Ecuador}

Indiferente del lugar, la responsabilidad es importante en la sociedad empresarial. En ese sentido, las entidades cumplen un papel fundamental en las organización de la colectividad. En los últimos años, la agenda de Ecuador en cuanto a la responsabilidad social empresarial (RSE), ha sido muy dinámica e interactiva debido al despertar del interés empresario frente a la innovación, sujetos a diferentes acciones de principio tales como: los congresos, los debates, los servicios profesionales de consultoría y sobre todo el más importante la sostenibilidad (Morán, Rodríguez, Torres, Aguilar, \& Villalta, 2016).

En Ecuador, lo primero que se llevó a cabo fue un estudio por organizaciones promotoras, tales como: IDE Business School, Fundación Avina, Cooperación Alemana al Desarrollo GIZ, Ekos negocios, entre otras. El estudio estuvo enfocado en la perspectiva de la responsabilidad social en los negocios, estas obtuvieron como resultado que los consumidores de la innovación tienen escasa información del tema, pero sí conocen que las empresas socialmente responsables son de gran importancia para el consumidor. Específicamente, el $86 \%$ considera que la responsabilidad social empresarial debe ser aplicada de manera inevitable en las organizaciones, además de ser controladas por el estado para así conservar el impulso del crecimiento económico. 
$\mathrm{Al}$ respecto, Ecuador forma parte de una red de estudio de las organizaciones promotoras del RSE en América Latina. Como resultado ha establecido que las empresas nacionales tornen sus ejes de acción del desarrollo sostenible. Por ejemplo, se tiene a la Cervecería nacional, la cual ha puesto su funcionamiento a favor del desarrollo sostenible. Esta es una empresa de 127 años de trayectoria en la industria de las bebidas alcohólicas con un amplio listado de productos en el mercado nacional e internacional. La cervecería pone atención a cinco prioridades del desarrollo sostenible con un alto compromiso que son: la cultura de moderación del consumo cervecero, el cuidado y desarrollo de la comunidad para mejorar los niveles de vida, la protección del medio ambiente, los procesos productivos de clase mundial y el mundo productivo con el uso mesurado de los recursos (Cervecería Nacional, 2014).

Es así como la Cervecería Nacional ha logrado como cometido que es posible sobresalir en las instituciones haciendo lo correcto y siendo empresas socialmente responsables, esto crea crecimiento económico por la buena acogida de las personas consumistas del producto.

No obstante, también hay empresas pequeñas y medianas en el mercado ecuatoriano que son un buen ejemplo de aplicación de la RSE en la innovación social (Charly \& Esteban, 2017). La clasificación de estas se presenta en la tabla 4.

\section{TABLA 4}

\section{Tipos de empresa}

\begin{tabular}{ll}
\hline \multicolumn{1}{c}{ Empresa } & \multicolumn{1}{c}{ Definición } \\
\hline $\begin{array}{l}\text { Empresa de Base } \\
\text { Tecnológica (EBT) }\end{array}$ & $\begin{array}{l}\text { Venta de propios } \\
\text { independientes centrales en el } \\
\text { aprovechamiento de un } \\
\text { proyecto o innovación } \\
\text { tecnológica. }\end{array}$ \\
& $\begin{array}{l}\text { Empresas principiantes que } \\
\text { están en proceso de } \\
\text { incorporación al mercado. } \\
\text { Start Up }\end{array}$ \\
& $\begin{array}{l}\text { Se da su origen en la } \\
\text { oportunidad de una empresa } \\
\text { matriz (sucursal). }\end{array}$ \\
\hline
\end{tabular}

Fuente: Charly \& Esteban (2017)
Cabe mencionar que el éxito de estas empresas podría deberse al tipo de actividad que realizan. Pues, un estudio realizado en Salinas de Guaranda demostró que las actividades que mayor ingreso tienen son la agricultura y la ganadería, estas generan otras actividades que, a su vez, son necesarias y tienen buena acogida (Barragán \& Ayaviri, 2017).

\section{Proyectos de innovación social en Ecuador}

Con el aumento de innovaciones sociales, se han originado financiamientos para emprendedores dando como resultados nuevos negocios sujetos en una serie de cambios, especialmente, en la tecnología e innovación social. Al respecto, según Lasio y Zambrano (2016), en Ecuador la inversión requerida para iniciar un proyecto es de $\$ 2.000$, lo cual se ilustra en el apéndice $\mathrm{A}$.

Según Altamirano, Bautista \& Tisalema (2019), las fuentes de financiamientos pueden variar según el emprendedor y estas pueden ser: con fondos propios, inversionistas informales, el gobierno, el capital de riesgo, crowdfunding, entre otras. En Ecuador la fuente mayoritaria son los fondos propios de los emprendedores, estos emprendedores son adultos en la mayoría, ya que se les facilita usar sus propios fondos para preparar el proyecto. Mientras que la fuente menos utilizada es el crowdfunding, dado que es una alternativa nueva que no genera mucha confianza. En Ecuador apenas se está entrenando en contexto y su uso es del $1 \%$ (ver apéndice B).

\section{CONCLUSIONES}

En Ecuador hay una estrecha relación entre los esfuerzos de desarrollar la innovación social y el emprendimiento. Las universidades son usadas como medio para el perfeccionamiento de la innovación social de los países, porque son la fuente de la perfección para las sociedades. Además, son las encargadas de educar a personas en las áreas que desean trabajar e invertir en un futuro, e iniciar proyectos empresariales para aumentar el desarrollo económico del país.

Para el desarrollo socioeconómico se encuentra en conjunto la tecnología y la 
innovación. La innovación, por su parte, busca aplicar conocimientos tecnológicos y es por esto por lo que siempre se debe actualizarlos mediante redes de conocimiento e invertir más en la educación de la población ecuatoriana. Solo así se formarán personas capaces de afrontar los cambios dados por las innovaciones y activos con una finalidad predispuesta a mantenerse en la inversión de un emprendimiento novedosos que llamen la atención de los consumidores y sean sostenibles en el tiempo (López \& Morales, 2019).

En el contexto ecuatoriano hay varios tipos de espacios en los que emprender sería una decisión correcta, ya que estos alcanzarían altos ingresos si van sujetos a actos innovadores y son: la educación, la salud y la producción, entre otros. Al respecto, Robalino (2016) menciona que estos deben ser trabajados de forma individual para observar el desempeño de cada uno de ellos y cómo mejoraría la productividad.

En otro aspecto, en Ecuador, todo lo que tenga que ver con el medio ambiente, no tiene una cultura que la priorice. Por ende, la innovación en este sector es efímera, porque las personas piensan que las empresas no medirán su impacto ambiental y solo le importará recoger el dinero de los cultivos o de los productos elaborados a base de esa materia prima obtenida del suelo (Robalino, 2016).

Cabe mencionar que, tanto en la tecnología como la agricultura se encuentran inmersas muchas personas $u$ organizaciones trabajando en ellas, pero las que más sobresalen son aquellas en las que su enfoque clave es la innovación. Sin embargo, descartando a las empresas no innovadoras, se mantiene un número alto de empresas que se desarrollan en los campos mencionados anteriormente, los cuales han servido para realizar un estudio sobre la actividad emprendedora y la competitividad en Ecuador (Mera, Burbano, \& Carrillo, 2018).

Por todo aquello, para lograr una innovación social sostenible en Ecuador, necesariamente los involucrados deben ser responsables socialmente, estar capacitados en el manejo de su área de negocio y ser emprendedores competitivos. Ya que, en muchos países en donde se tiene una actitud emprendedora por parte de sus ciudadanos existe un dinamismo empresarial que crea economías fuertes.

Respecto a la competitividad en el ámbito de la innovación social, esta refiere a la capacidad de una nación en sostener sus proyectos e incrementarlos hasta llegar a mercados internacionales. En el caso de Ecuador, sus habitantes son personas muy emprendedoras, pero lastimosamente no son sostenibles, ya que no cuentan con principios que las ayuden a mantenerse. Dado aquello, la escaza productividad que se encuentra en los emprendimientos produce economías precarias, lo cual es muy característico de países en vías de desarrollo. Sin embargo, una mejora en la competitividad ha sido generada gracias a las actitudes emprendedoras de la población ecuatoriana, las cuales se ilustran en el apéndice C.

Las habilidades emprendedoras integradas por los pilares mostrados en el apéndice C, fundamentales para emprendimientos, son espacios donde hay oportunidad de emprender. En Ecuador esto ha sido aprovechado por los ciudadanos para iniciar nuevos negocios y así avanzar en la vertiente económica en la que la motivación es la competitividad.

Para Dainienè y Dagilienè (2015), las innovaciones sociales tienen que satisfacer necesidades sociales en plazos de ocupación, salud, educación, etc. Por ello, deben tener ciertos puntos para ser factibles y estos son:

- Tener algunos elementos novedosos o nuevos en su contexto,

- Tiene que ser implementado,

- Y tienen que ser eficaces.

Implementados de forma correcta pueden hacer que las empresas aquí en Ecuador alcancen el mercado internacional y no basarse únicamente en publicidad reflexiva o campañas de ayuda social (Dziallasun \& Cegarun). Por tanto, los directores, gerentes o profesionales encargados 
del área de desarrollo e investigación de las empresas, deben enfocar sus esfuerzos en establecer políticas y procesos orientados a materializar la responsabilidad social para alcanzar la innovación. Mientras que los propietarios de micro, pequeñas y medianas empresas deben producir o comercializar o prestar sus bienes y servicios considerando los mercados en donde puedan tener mayor impacto.

\section{REFERENCIAS}

Altamirano, D., Bautista, P., \& Tisalema, J. (2019). Gobierno cooperativo e innovación social en el sector de la economía popular y solidaria. Dialnet, 6(1), 24-48.

Álvarez, M., \& Macías, M. (2015). Innovación y creatividad como motores de desarrollo y el caso de las empresas de Guayaquil (Ecuador). Economía Pública, Social y Cooperativa, 85, 4-22. doi:0.7203

Ávila, R., \& Campos, L. (2018). La economía social ante los paradigmas económicos emergentes: innovaciónsocial, economía colaborativa, economíacircular, responsabilidad socialempresarial, economía del bien común,empresa social y economía solidaria. Revista de Economía Pública y Social, 93, 5-50. doi:10.7203/CIRIEC-E.93.12901

Barragán, C., \& Ayaviri, V. (2017). Innovación y Emprendimiento y su relación con el Desarrollo. Información Tecnológica, 28(6), 71-80. doi:10.4067/S071807642017000600009

Blanco, I., Gallach, H., \& Martínez, R. (2015). El papel de la innovación social. Universitat Autónoma de Barcelona.

Calderón, A. (2015). Situacion de la educacion rural en Ecuador. RIMSIP. Centro latinoamericano para el desarrollo rural.

Charly, C., \& Esteban, A. (2017). Entrepreneurship and innovation in Ecuador, analysis of business ecosystems for the consolidation of small and medium enterprises. INNOVA Research Journal, 2(9.1), 73-87. doi:https://doi.org/10.33890/innova.v2.n9 .1.2017.504

Christensen, C., Baumann, H., Ruggles, R., \& Sadtler, T. (2006). Disruptive innovation for social change. Harvard Business review, 84(12), 73-87.

Dainiené, R., \& Dagiliené, L. (2015). Approach Based Theoretical Framework for Measuring Social Innovations. ProcediaSocial and behaviors sciences, 213, 275280. doi:10.1016/j.sbspro.2015.11.537

Dziallasun, M., \& Cegarun, K. (s.f.). The Innovation.

Echeverria, J. (2008). The Oslo Manual and The Social Innovation. Arbor. Ciencia, Pensamiento y Cultura, 609-618. doi:0210-1963

Estrada, N. (2014). Discusión del término "innovación social": ambigüedad del término y elementos para una propuesta de definición estipulativa. Medellín: Universidad EAFIT.

Fantova, F. (2018). Contruyendo la intervención social. Papeles del Psicólogo, 39(2).

Goleman, D. (2018). Inteligencia emocional en las empresas. Penguin Random House.

Hernández, J., Tirado, P., \& Ariza, A. (2016). El concepto de innovación social: ámbitos, definiciones, alcances teóricos. Revista de economía pública, social y cooperativa, 88, 165-199.

Hochgerner, J., Franz, H., \& Howaldt, J. (2011). Vienna declaration: the most needed social innovations and related research topics. Challenge social innovation, 1921.

Howaldt, J. (2016). La innovación social: hacia un nuevo paradigma (Vol. 1). (D. 
Domanski, N. Monge, G. Quitiaquez, \& D. Rocha, Edits.) UNIMINUTO.

Hubert, A. (2011). Empowering people, driving change: Social innovation in the European Union. European Union.

Lasio, V., \& Zambrano, J. (2016). Financiamiento para emprendedores. Escuela Superior Politécnica del Litoral, Guayaquil.

López, A. (2016). Propuesta de modelo de evaluación de la innovación social universitaria responsable. Estudios sobre educación, 30, 71-93. doi:10.1558/004.30.71-93

López, A., \& Morales, V. (2019). Development and innovation processes: application of a methodology to. Brazilian Journal of Development. Brazilian Journal of Development, 5(6), 4550-4567.

Lozano, M., Mendoza, M., Felipe, R., \& Welter, Z. (2016). La apropiación social de la ciencia, la tecnología y la innovación. Políticas y prácticas en Chile, Colombia, Ecuador y Perú. Ciencia, tecnología y sociedad, 8(15), 25-49. doi:2145- 4426

Marin, E. (2018). La gestión de las emociones. Inteligencia Artificial. Madrid: Elearning S.L.

Marín, V., \& Cabero, J. (2019). Social networks in education: from innovation to educational research. Revista Iberoamericana de Educación a Distancia, 22(2), 22-33. doi:10.5944/ried.22.2.24248

Martínez, D., González, N., \& Nieto, M. (2015). La innovación social como motor de creación de las empresas. Universia Business Review, 67, 48-63.

Mazini, E. (2015). Design, when everybody designs: An introduction to design for social innovation. Cambrigde: The Mit Press.
Mendez, R. (2001). Innovación y redes de cooperación para el desarrollo local. Revista Internacional de desenvolvimiento local, 2(3), 37-44.

Mera, B., Burbano, G., \& Carrillo, A. (2018). Actividad emprendedora y competitividad en Ecuador. Revista Global de Negocios, 6(1), 29-44.

Moliner, J., Azorín, J., Guilló, J., Ortega, E., \& Gamero, M. (2019). Sostenibilidad, calidad, innovación y ventaja competitiva en el sector hotelero. Revista Internacional de Turismo, Empresa y Territorio, 1-21.

Mora, M., \& Martínez, R. (2018). Desarrollo local sostenible, responsabilidad social corporativa y emprendimiento social. Dialnet, 31, 27-46. doi:http://dx.doi.org/10.19052/ed.4375

Morán, C., Rodríguez, L., Torres, M., Aguilar, A., \& Villalta, M. (2016). Atakeholders, responsabilidad social en Ecuador. Revista Científica y Tecnológica, 3(2), 21-30.

Nieto, M., \& Gómez, R. (2004). Responsabilidad social corporativa: la última innovación. Management, 28-39. doi:1698-5117

Ogburn, W. (1939). Social change. New York: Huebsch.

Ortíz, N. (2016). Diseñando el cambio. La innovacion social y sus retos. Economía Creativa, 9-35.

Orué-Arias, E. (2017). Inteligencia emocional y desempeño laboral de los trabajadores de una empresa peruana. Revista de Ciencias Empresariales de la Universidad de San Martín, 3(1), 79-95.

Robalino, A. (2016). CO2 emissions convergence among 10 South American countries. A study of Kayacomponent. Carbon Management, 7, 1-12. 
doi:https://doi.org/10.1080/17583004.201 6.1151502

Rossi, J. (2016). La innovación social en Commons. Análisis Económico, 31(76), 141-164.

S.A., C. N. (2014). Cervecería Nacional. Obtenido de https:// www. cervecerianacional.ec/desarrollosostenible.php

Serrano, M., Hervás, J., \& Campos, C. (2017). Influencia de la experiencia laboral y del entorno social próximo en las intenciones de emprender de los estudiantes de ciencias de la actividad física y el deporte. Sports Economics \& Management, 7(1), 14-29.

Tortosa, J. (2018). Innovación social como motor del desarrollo local y el emprendimiento social. Dialnet, 31(20), 11-25.

doi:http://dx.doi.org/10.19052/ed.4416 


\section{APÉNDICES}

\section{Apéndice A}

\section{Financiamiento para emprendedores}

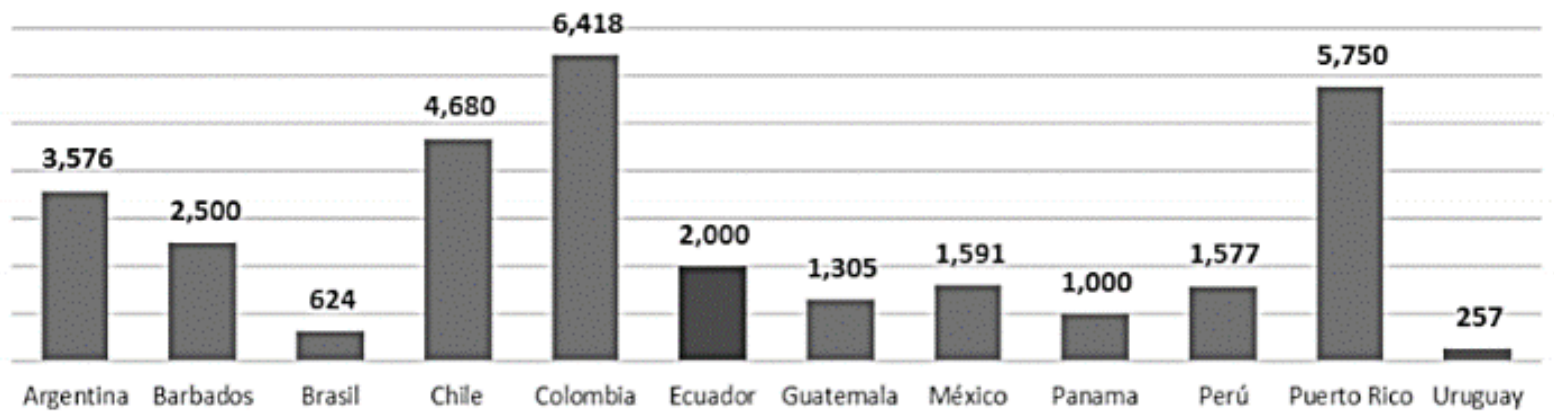

Fuente: Lasio y Zambrano (2016)

\section{Apéndice B}

\section{Tipos de Financiamiento para emprendedores}

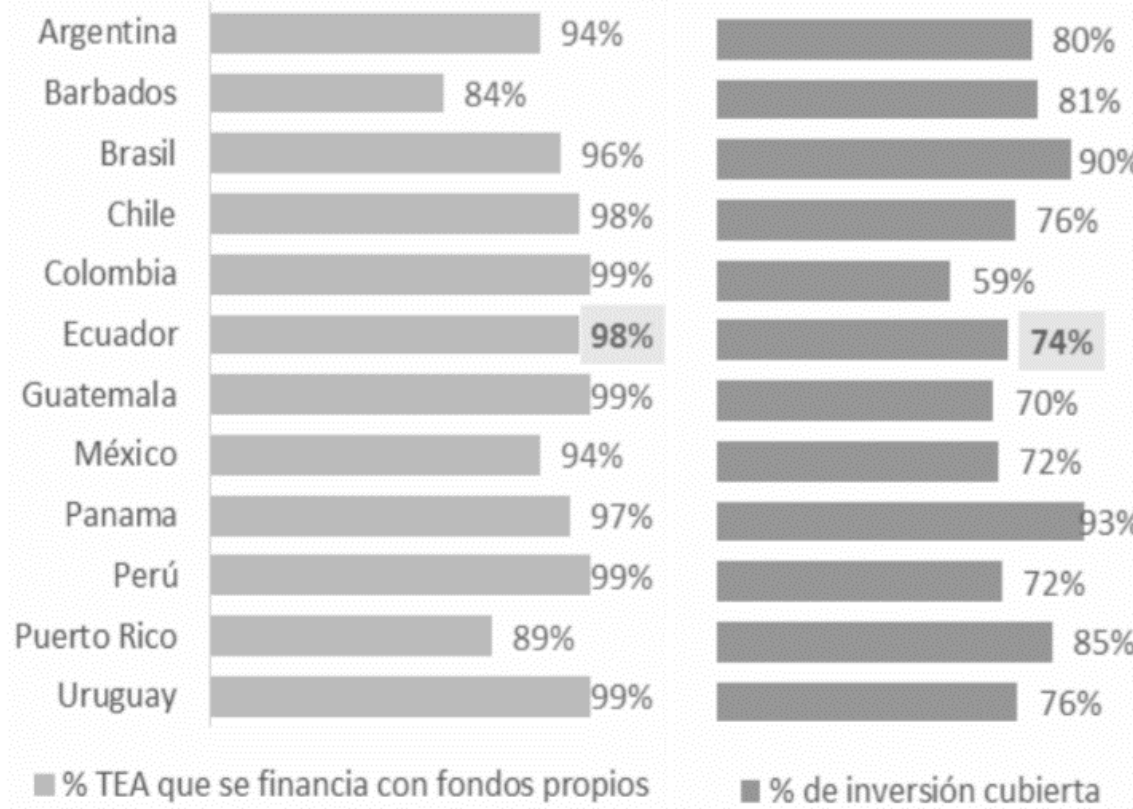

Fuente: Lasio y Zambrano (2016) 


\section{Apéndice C}

\section{Actitudes emprendedoras}

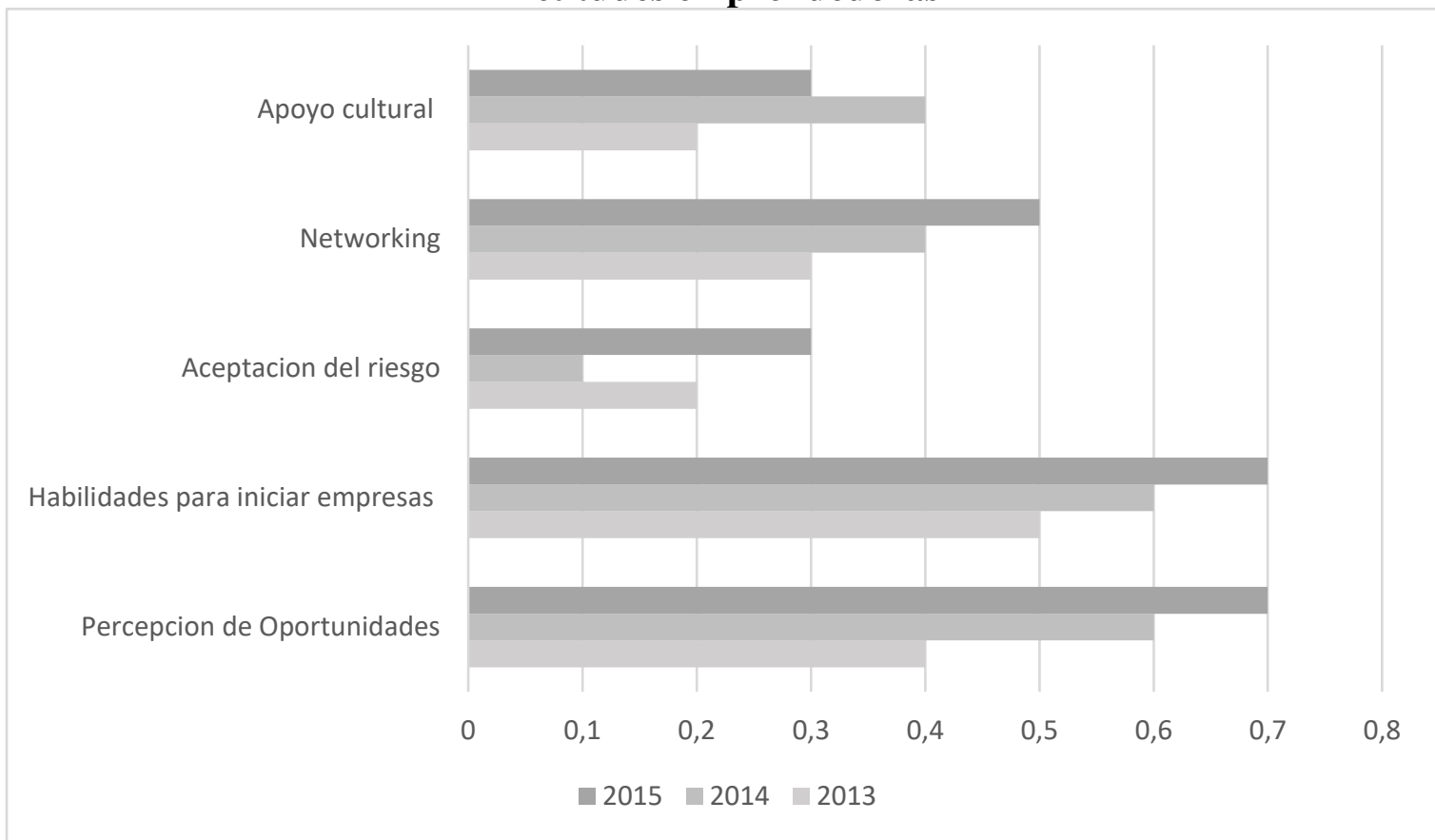

Fuente: Mera, Burbano y Carrillo (2018) 